5. Mollon J, David AS, Morgan C, et al. Psychotic experiences and neuropsychological functioning in a population-based sample. JAMA Psychiatry. 2016;73(2):129-138.

6. Gur RC. Prospective community studies linking cognitive deficits to subclinical symptoms and a step toward precision medicine. JAMA Psychiatry. 2016;73(2):109-110.

\section{How Similar Are the Disorders Included Under the Umbrella of Obsessive-Compulsive Disorder and Related Disorders?}

To the Editor In JAMA Psychiatry, Grant and colleagues ${ }^{1}$ demonstrated the efficacy of $\mathrm{N}$-acetylcysteine (NAC) in the treatment of excoriation disorder (ED). The same group had previously reported the benefits of NAC for adults with trichotillomania (TTM). ${ }^{2}$ In both studies, NAC was used in monotherapy in otherwise treatment-free patients. In the $D S M-5$, ED and TTM were included under the umbrella of obsessive-compulsive disorder (OCD) and related disorders because of similarities regarding phenotypic expression and common putative neurobiological underpinnings between these disorders and OCD. When used for treatment-resistant OCD, NAC was not more effective than placebo as a selective serotonin reuptake inhibitor (SSRI) augmentation strategy. ${ }^{3}$

In striking contrast with what we know about OCD, SSRIs have not been proved to be superior to placebo in TTM treatment. ${ }^{2}$ As for ED, the evidence regarding the efficacy of SSRIs is still scarce. ${ }^{1}$ So far, there are no US Food and Drug Administration-approved treatments for ED and TTM. The divergent results of the trials testing either SSRIs or NAC for ED/ TTM and OCD suggest that treatments may not be used interchangeably between OCD and related disorders. Despite the possible methodological issues related to the design of specific trials, do such heterogeneous results regarding treatment response relate to heterogeneous neurobiology between OCD and related disorders?

Pathological grooming behaviors in animals are widely used in the literature as a common animal model of ED/TTM and OCD. However, findings derived from animal studies do not always confirm the association between grooming behavior and OCD. For example, no differences were observed in the results of the marble-burying test of rats with different levels of grooming behavior. ${ }^{4}$

In addition, results from genetic studies also suggest that the neurobiology of pathological grooming may not be necessarily related to OCD. It had been demonstrated that Sapap3 knockout mice groomed themselves excessively. However, using family-based association analyses, Bienvenu et $\mathrm{al}^{5}$ found that variation within the human equivalent of the Sapap3 gene appeared associated with ED/TTM, but not with OCD.

The conflicting results regarding the efficacy of NAC for ED/TTM and OCD, in addition to the findings of translational studies, suggest that the neural basis of repetitive behaviors observed in patients with these disorders might not be the same. So far, similarities between ED/TTM and OCD do not seem to go beyond the characterization by repetitive behaviors and some degree of familial aggregation.
Daniel L. C. Costa, MD

Juliana Belo Diniz, MD, PhD

Eurípedes Constantino Miguel, MD, PhD

Author Affiliations: Department and Institute of Psychiatry, University of Sao Paulo Medical School, Sao Paulo, Brazil.

Corresponding Author: Eurípedes Constantino Miguel, PhD, Department and Institute of Psychiatry, University of Sao Paulo Medical School, R Dr Ovídio Pires de Campos, 785. 05403-010 SP, Brazil (ecmiguel@usp.br).

Published Online: July 13, 2016. doi:10.1001/jamapsychiatry.2016.1342.

Conflict of Interest Disclosures: None reported.

1. Grant JE, Chamberlain SR, Redden SA, Leppink EW, Odlaug BL, Kim SW. $\mathrm{N}$-acetylcysteine in the treatment of excoriation disorder: a randomized clinical trial. JAMA Psychiatry. 2016;73(5):490-496.

2. Grant JE, Odlaug BL, Kim SW. $\mathrm{N}$-acetylcysteine, a glutamate modulator, in the treatment of trichotillomania: a double-blind, placebo-controlled study. Arch Gen Psychiatry. 2009;66(7):756-763.

3. Costa DLC, Diniz JB, Joaquim M, et al. Serotonin reuptake inhibitor augmentation with $\mathrm{N}$-acetylcysteine in treatment-resistant obsessivecompulsive disorder: a double-blind randomized controlled trial. Biol Psychiatry. 2015;77(9):63S.

4. Reimer $A E$, de Oliveira AR, Diniz JB, Hoexter MQ, Chiavegatto S, Brandão $M L$. Rats with differential self-grooming expression in the elevated plus-maze do not differ in anxiety-related behaviors. Behav Brain Res. 2015;292:370-380.

5. Bienvenu OJ, Wang Y, Shugart YY, et al. Sapap3 and pathological grooming in humans: results from the OCD Collaborative Genetics Study. Am J Med Genet B Neuropsychiatr Genet. 2009;150B(5):710-720.

In Reply Costa and colleagues raise important issues regarding how grooming disorders (GDs) should be optimally treated and classified.

$\mathrm{N}$-acetylcysteine (NAC) showed superiority over placebo as monotherapy in excoriation disorder, ${ }^{1}$ similar to findings in trichotillomania. However, NAC's findings in obsessivecompulsive disorder (OCD) are mixed. Two clinical trials reported significant benefits of NAC augmentation over placebo for OCD, ${ }^{2,3}$ while a third study did not show benefits over placebo. ${ }^{4}$ As Costa and colleagues suggest, differential pharmacological response across disorders is an important factor when considering diagnostic classification. This is also complicated by differences in study designs, as results may vary when using NAC as augmentation to a selective serotonin reuptake inhibitor rather than a primary intervention.

A common link between GD and OCD is an appealing notion based on overt symptoms: these conditions are characterized by repetitive acts that are difficult to suppress. There is evidence for familial and comorbid overlap as well, but whether this overlap is stronger than between GD and other disorders (eg, alcoholism or attention-deficit/hyperactivity disorder) is less clear. Neuropsychological deficits in OCD span a broader range of domains than those identified in GD. Even without these differences, the neurobiological underpinnings may differ between disorders. Also, cognitive dysfunction is heterogeneous even within a given disorder: while patient groups may manifest general cognitive deficits relative to control groups, these problems may not always persist when assessed on an individual basis. The logical question then is whether cognitive, imaging, or other markers could be used to individualize treatment based on unique clinical features, possibly transdiagnostically. 
We agree with the caveats by Costa et al regarding animal models of GD and OCD. In our experience, some animal models of OCD are in fact measuring excessive grooming, while other models of excessive grooming bear little face validity with human disorders, such as animals who excessively groom cage-mates, as opposed to themselves. While these modalities may be beneficial in assessing wider trends, their utility in optimizing treatment based on individual traits may be more limited.

We suggest that current diagnostic classification of GD is arbitrary but pragmatic given available evidence. Classifying GD with OCD in the DSM-5 encourages clinicians to screen for relevant comorbidities and encourages research to support or refute this conceptualization. Whether the International Statistical Classification of Diseases and Related Health Problems, Eleventh Revision will adopt a similar classification remains unclear because GDs are currently listed in a separate category to OCD in the International Statistical Classification of Diseases and Related Health Problems, Tenth Revision. Despite possible neurobiological or cognitive distinctions, it may be beneficial to classify GD and OCD under a common heading based on similar symptoms, given that not all clinicians have experience with GD and may conflate symptoms with OCD unless distinct guidelines are provided, as is often the case with disorders falling under a common classification.

Also of relevance here is the concept of behavioral addiction. In an animal model, NAC dampened the effects of cocaine on glutamate transporters in the nucleus accumbens and dorsal striatum. ${ }^{5}$ Perhaps the beneficial effects of NAC in GD occur at least partly via a similar mechanism, an issue that could be explored further.

\section{Eric W. Leppink, BA \\ Samuel R. Chamberlain, MD, PhD \\ Jon E. Grant, JD, MD, MPH}

Author Affiliations: Department of Psychiatry and Behavioral Neuroscience, Pritzker School of Medicine, University of Chicago, Chicago, Illinois (Leppink, Grant); Department of Psychiatry, University of Cambridge, Cambridge, England (Chamberlain); Cambridge and Peterborough NHS Foundation Trust, Cambridge, England (Chamberlain)
Corresponding Author: Jon E. Grant, JD, MD, MPH, Department of Psychiatry and Behavioral Neuroscience, Pritzker School of Medicine, University of Chicago, 5841 S Maryland Ave, MC 3077, Chicago, IL 60637 (jongrant@uchicago .edu).

Published Online: July 13, 2016. doi:10.1001/jamapsychiatry.2016.1452. Conflict of Interest Disclosures: Dr Chamberlain reported being a paid consultant for Cambridge Cognition, and his involvement was facilitated by a grant from the Academy of Medical Sciences. Dr Grant reported receiving grant U01MH076179 from the National Institute of Mental Health and research grants from the National Center for Responsible Gaming, Forest Pharmaceuticals, and Roche Pharmaceuticals; yearly compensation from Springer Publishing for acting as editor in chief of the Journal of Gambling Studies; and royalties from Oxford University Press, American Psychiatric Publishing Inc, Norton Press, and McGraw Hill. No other disclosures were reported.

1. Grant JE, Chamberlain SR, Redden SA, Leppink EW, Odlaug BL, Kim SW. $\mathrm{N}$-acetylcysteine in the treatment of excoriation disorder: a randomized clinical trial. JAMA Psychiatry. 2016;73(5):490-496.

2. Afshar $\mathrm{H}$, Roohafza $\mathrm{H}$, Mohammad-Beigi $\mathrm{H}$, et al. $\mathrm{N}$-acetylcysteine add-on treatment in refractory obsessive-compulsive disorder: a randomized, double-blind, placebo-controlled trial. J Clin Psychopharmacol. 2012;32(6):797 803.

3. Paydary K, Akamaloo A, Ahmadipour A, Pishgar F, Emamzadehfard S, Akhondzadeh S. N-acetylcysteine augmentation therapy for moderate-tosevere obsessive-compulsive disorder: randomized, double-blind placebo-controlled trial. J Clin Pharm Ther. 2016;41(2):214-219.

4. Sarris J, Oliver G, Camfield DA, et al. N-acetyl cysteine (NAC) in the treatment of obsessive-compulsive disorder: a 16-week, double-blind, randomised, placebo-controlled study. CNS Drugs. 2015;29(9):801-809.

5. Ducret $E$, Puaud M, Lacoste J, et al. N-acetylcysteine facilitates self-imposed abstinence after escalation of cocaine intake [published online October 8 , 2015]. Biol Psychiatry. doi:10.1016/j.biopsych.2015.09.019.

\section{CORRECTION}

Omitted Statistical Analysis Description and Errors in Figure: In the Original Investigation titled "Whole-Body Hyperthermia for the Treatment of Major Depressive Disorder: A Randomized Clinical Trial,"1 published online May 12, 2016, there was an omission in the Methods section and errors in Figure 2. The sentence describing the Cohen $d$ calculation in the Statistical Analysis subsection of the Methods section should have read as follows: "Cohen $d$ was calculated to assess effect sizes for between-group differences at all posttreatment points based on means and SDs derived from the mixed-effect model." There were also data errors in Figure 2, which have been corrected, and the accompanying figure caption should have read as follows: "The error bars indicate SEs. Means and SEs were derived from the raw data provided in Table 2." This article was corrected online.

1. Janssen CW, Lowry CA, Mehl MR, et al. Whole-body hyperthermia for the treatment of major depressive disorder: a randomized clinical trial [published online May 12, 2016]. JAMA Psychiatry. doi:10.1001/jamapsychiatry.2016.1031. 